G. Stroica (Saint John)

\title{
A NATURAL CHARACTERIZATION OF NONLINEAR COST RULES
}

Abstract. We prove a new characterization of cost rules based on the relationship between the classes of unambiguous and nonwasteful assets in incomplete frictionless markets.

1. Introduction. The no-arbitrage principle together with the assumption of complete markets enforce linear cost rules (see e.g. [1]). In incomplete markets, where not all securities can be replicated by feasible portfolios, it is possible to stay on the safe side only by superhedging strategies. In this latter case, one needs a cost function to determine the minimum value necessary for the replication or superreplication of any contingent claim; however, the standard linear approach fails for any nonattainable claim. Instead, the following nonlinear set-up is commonly employed (cf. [9], [4], 7], 10]). The space of financial positions is a vector space $E$ with vector ordering $\geq$; besides the origin $\mathbf{0} \in E$, we distinguish a reference cash stream $\mathbf{1}>\mathbf{0}$ (by definition, $x>\mathbf{0}$ means $x \geq \mathbf{0}$ and $x \neq 0$ ). A real-valued cost function $C$ is defined on $E$ through the following properties (cf. [4):

- Subadditivity: $C(x+y) \leq C(x)+C(y)$ for any $x, y \in E$;

- Positive homogeneity: $C(t x)=t C(x)$ for any nonnegative real $t$ and $x \in E$;

- Monotonicity: $C(x) \leq C(y)$ if $x \leq y$ in $E$;

- Translation invariance: $C(x+t \mathbf{1})=C(x)+t$ for any real $t$ and $x \in E$.

In the space of linear price systems $E^{\prime}$, the algebraic dual of $E$, we fix a total subspace $E^{\times}$(i.e., if $g(x)=0$ for all $g \in E^{\times}$, then $x=\mathbf{0}$ ), and consider the weak ${ }^{*}$-topology on $E^{\times}$associated to the dual pair $\left(E, E^{\times}\right)$. We say that $g \in E^{\times}$is positive (resp. normalized) if $g(x) \geq 0$ for $x \geq \mathbf{0}$ in $E$ (resp.

2010 Mathematics Subject Classification: Primary 91B24; Secondary 54F05.

Key words and phrases: cost rules, unambiguous assets, nonwasteful assets, incomplete frictionless market, relevance, order convergence topology. 
$g(\mathbf{1})=1$ ). In this general set-up, we have the following structure result (cf. [9], [4], 7], [10]): any cost function $C$ on $E$ is of the form

$$
C(x)=\sup _{g \in \mathcal{A}} g(x)
$$

for some weak ${ }^{*}$-closed convex set $\mathcal{A} \subseteq E^{\times}$in which all $g \in \mathcal{A}$ are positive and normalized. The proof relies on the fact that $\mathcal{A}$ consists of all positive and normalized elements of the polar set of $\{x \in E ; C(-x) \leq 0\}$ with respect to the dual pair $\left(E, E^{\times}\right)$, i.e.,

$$
\begin{aligned}
& \mathcal{A}=\left\{g \in E^{\times} ; g\right. \text { is normalized and } \\
& \qquad g(x) \geq 0 \text { for any } x \text { satisfying } C(-x) \leq 0\} .
\end{aligned}
$$

In this paper we study the possibility of replacing the set $\mathcal{A}$ by a less abstract one; this will be accomplished when the set of nonambiguous assets coincides with the set of nonwasteful assets in incomplete frictionless markets, and under minimal regularity requirements on the cost function (i.e., relevance and continuity). As such, the sup in formula (1) can be taken upon the set of functionals that agree on all nonambiguous assets; this is very natural, as the space of unambiguous assets equals the space of attainable claims, in finite dimensions, but also in special cases of infinite-dimensional markets (cf. [1]).

\section{Main result and examples}

Definitions (cf. [10], 4], [2], [11]). Let $f_{0} \in E^{\prime}$. We say that the cost function $C$ is $f_{0}$-relevant if

$$
x \geq \mathbf{0} \text { and } f_{0}(x)>0 \text { imply } C(x)>0 .
$$

To a cost function $C$, we associate the set of unambiguous assets $U A_{C}$ by

$$
U A_{C}=\{x \in E ; C(x)+C(-x)=0\} .
$$

If, in addition, $C$ is $f_{0}$-relevant, we introduce the set of nonwasteful assets $N W_{C}$ by:

$$
N W_{C}=\left\{x \in E ; C(y)>C(x) \text { provided } y \geq x \text { and } f_{0}(y)>f_{0}(x)\right\} .
$$

We say that the sequence $\left(x_{n}\right)_{n \geq 1}$ in a vector space with vector ordering $E$ is order convergent, or (o)-convergent, and write $x=(\mathrm{o})-\lim x_{n}$, if there exist two sequences $a_{n}$ increasing to $\mathbf{0}$ and $b_{n}$ decreasing to $\mathbf{0}$ in $E$ such that $a_{n} \leq$ $x_{n}-x \leq b_{n}$ for all $n$. We say that the cost function $C$ is order continuous, for short (o)-continuous, if $C\left(x_{n}\right) \rightarrow C(x)$ provided $x=(\mathrm{o})$ - $\lim x_{n}$.

Interpretation. The relevance property ensures that the cost function identifies positions with strictly positive cost: each position with $x \geq \mathbf{0}$ satisfies $f_{0}(x) \geq 0$, but only those satisfying $f_{0}(x)>0$ have strictly positive cost. Note that the definitions for relevance introduced in [9] and [3] are 
particular cases of (3). The set $U A_{C}$ describes the assets for which there is no pricing distinction between a selling position and a buying position. The set $N W_{C}$ describes the claims with the following property: if some payoff assigned by the claim is replaced by a better payoff, i.e., $f_{0}(x)>f_{0}(y)$, then the resulting contingent claim $C(y)$ is strictly more expensive than the original one $C(x)$.

Our main result below concerns the possibility of replacing the set $\mathcal{A}$ in formula (1) by the set $\mathcal{B}$ defined as follows:

$$
\begin{aligned}
& \mathcal{B}=\left\{g \in E^{\times}: g\right. \text { is positive, normalized and } \\
& \left.\qquad g(x)=C(x) \text { for any } x \in U A_{C}\right\} .
\end{aligned}
$$

Our result reads as follows (note that it extends the - simpler - finite dimension version of the main result in [2]):

THEOREM. Let $C$ be an $f_{0}$-relevant $(\mathrm{o})$-continuous cost function and $E^{\times}$ a Banach subspace of $E^{\prime}$. Then $\mathcal{B}=\mathcal{A}$ if and only if $N W_{C}=U A_{C}$.

EXAMPLE 1 (concerning the general setup). Consider a probability space $(\Omega, \mathcal{F}, P)$, and define $\mathbf{1}(\omega)=1$ a.e. One can take:

- $E=\mathbf{R}^{\Omega}$, the space of real-valued functions on a finite set $\Omega$, with the pointwise ordering, and where $E^{\times}$is the space of all probability measures on $\Omega$ (see [3], [5]);

- $E=L^{p}(\Omega, \mathcal{F}, P), 1 \leq p \leq \infty$, with the pointwise $P$-a.e. ordering, and where $E^{\times}$is the norm topological dual of $E$ (see [4], [6]); if $p=\infty$, recall that $E^{\times}=\mathbf{b a}(\Omega, \mathcal{F}, P)$, the Banach space of bounded finitely additive measures on $\mathcal{F}$, absolutely continuous with respect to $P$.

Alternatively, $E$ can be the space of $p$-summable sequences $l^{p}(1 \leq p \leq \infty)$, the space of continuous functions $C(\Omega)$ on a compact Hausdorff space $\Omega$, a standard Lebesgue or Orlicz function space (see [4]), a space of test functions (e.g., $C^{\infty}$ with polynomial growth at infinity), etc. with their natural orderings and topologies.

EXAMPLE 2 (concerning $f_{0}$-relevance). Each nonnegative position $x \in$ $E=L^{\infty}(\Omega, \mathcal{F}, P)$ satisfying $P[x>0]>0$ has strictly positive cost (see [4, [10]). Also note that stricly positive cost functions are relevant with respect to any strictly positive linear functionals (such as strictly positive probabilities, e.g., atomless, or even probabilities that do not charge the states of the market). The following quantitative method shows how "relevant" a cost rule is. Consider the space of a.e. finite measurable functions $x$ with respect to the Lebesgue measure $\lambda$ on $(0, \infty)$ satisfying

$$
\lambda(t>0 ;|x(t)|>c) \rightarrow 0 \quad \text { when } c \rightarrow \infty .
$$

Endowed with convergence in measure and pointwise $\lambda$-a.e. ordering, this 
space becomes a nonseparable complete metric vector lattice, and any element $f_{0}$ of its topological dual can be written as follows:

$$
f_{0}(x)=\lim _{c \rightarrow \infty} \int \inf \{x, c\} d m_{f}
$$

for some finitely additive signed measure $m_{f}$ on the Lebesgue $\sigma$-field of $(0, \infty)$ satisfying $m_{f}(A)=0$ for any $A \subset(0, \infty)$ with $\lambda(A)<\infty$. Given some threshold $t_{0}>0$, the inequality $f_{0}(x) \leq t_{0}$ quantifies how relevant a cost, larger than $c$, is.

EXAMPLE 3 (concerning (o)-convergence and (o)-continuity). In the space $L^{1}(\Omega, \mathcal{F}, P)$, order convergence is equivalent to: $x_{n} \rightarrow x$ a.e. and $\left|x_{n}\right| \leq c$ a.e. for some constant $c>0$; in $L^{p}(\Omega, \mathcal{F}, P), 1 \leq p<\infty$, it is equivalent to: $x_{n} \rightarrow x$ a.e. and $\left|x_{n}\right| \leq y$ a.e. for some $y \in L^{p}$; in $L^{\infty}(\Omega, \mathcal{F}, P)$ it coincides with a.e. convergence. Continuity from below or lower semicontinuity on bounded ordered vector spaces implies (o)-continuity (see [10. p. 798]). If $E=L^{\infty}(\Omega, \mathcal{F}, P)$ and $E^{\times}=\mathbf{b a}(\Omega, \mathcal{F}, P)$, then any $f \in E^{\times}$ continuous from below is (o)-continuous, i.e., $\sigma$-additive.

EXAMPle 4 (a cost function for which $N W_{C}=U A_{C}$ ). Consider $E=$ $L^{\infty}(\Omega, \mathcal{F}, P)$, and define

$$
C(x)=\operatorname{ess} \sup x .
$$

In this case $E^{\times}=\mathcal{A}=L^{1}(\Omega, \mathcal{F}, P)$, the cost $C$ is (o)-continuous and relevant with respect to $P$, and both $N W_{C}$ and $U A_{C}$ are equal to the set of constant functions $P$-a.e. on $\Omega$.

ExAMPLE 5 (another cost function for which $N W_{C}=U A_{C}$ ). On $[0,1]$ endowed with the Borel $\sigma$-algebra $\mathcal{F}$, we define

$$
C(x)=\min \{m:\{x(\cdot)>m\} \text { is of first category }\} .
$$

In this case $\mathcal{A}$ is a convex set of purely finitely additive probabilities on $\mathcal{F}$, the $\operatorname{cost} C$ is (o)-continuous and relevant with respect to the Lebesgue measure on $[0,1]$, and both $N W_{C}$ and $U A_{C}$ are equal to the set of functions Lebesgue-a.e. constant on sets of first category in $[0,1]$.

EXAMPle 6 (a cost function for which $N W_{C} \neq U A_{C}$ ). Consider an atomless probability space $(\Omega, \mathcal{F}, P)$, fix $0<\alpha<1$, and define

$$
C(x)=\sup _{P(A)>\alpha} \int_{A} x d P .
$$

In this case $\mathcal{A}=\left\{x \geq 0 ;\|x\|_{\infty} \leq 1 / \alpha, \int x d P=1\right\}$, the cost $C$ is (o)continuous and relevant with respect to $P$, and $U A_{C}=L^{1}(\Omega, \mathcal{F}, P)$. However, the sum of an $L^{1}(\Omega, \mathcal{F}, P)$-function and the indicator of any $P$-negligible subset of $\Omega$ does not belong to $N W_{C}$. 
3. Proof of the main result. We collect the easy statements in the following lemma, and present the essential arguments of the proof in two propositions.

Lemma 1. Let $C$ be a cost function; then $\mathcal{A} \subseteq \mathcal{B}$. If, in addition, $C$ is $f_{0}$-relevant, then $U A_{C} \subseteq N W_{C}$.

Proof. For the first part, let $x \in E$ be such that $C(x)+C(-x)=0$. We then have

$$
\sup _{g \in \mathcal{A}} g(x)=-\sup _{g \in \mathcal{A}} g(-x)=\inf _{g \in \mathcal{A}} g(x),
$$

therefore $g(x)=C(x)$ for all $g \in \mathcal{A}$.

For the second part, let $x_{0} \in U A_{C}$. According to (4), (3) and using subadditivity, for $y \geq x_{0}$ satisfying $f_{0}(y)>f_{0}\left(x_{0}\right)$, we have

$$
C(y)-C\left(x_{0}\right)=C(y)+C\left(-x_{0}\right) \geq C\left(y-x_{0}\right)>0,
$$

so, by (5), we conclude that $x_{0} \in N W_{C}$.

Proposition 2. Let $C$ be an $f_{0}$-relevant cost function. Then $N W_{C} \subseteq$ $U A_{C}$ implies $\mathcal{B} \subseteq \mathcal{A}$.

Proof. Let $f \in \mathcal{B}$; if $f \notin \mathcal{A}$, according to (2) there exists $x_{0}$ with $C\left(-x_{0}\right) \leq 0$ and $f\left(x_{0}\right)<0$. According to (1), we deduce that $g\left(x_{0}\right) \geq 0$ for all $g \in \mathcal{A}$, so

$$
f\left(x_{0}\right)<0 \leq g\left(x_{0}\right) \quad \text { for all } g \in \mathcal{A} .
$$

Assume that for any $y \geq x_{0}$ with $f_{0}(y)>f_{0}\left(x_{0}\right)$ we have $C(y)>C\left(x_{0}\right)$. As $N W_{C} \subseteq U A_{C}$, using (4)-(5) we have $C\left(x_{0}\right)+C\left(-x_{0}\right)=0$, so, as in the proof of Lemma 1 (first part), we obtain $g\left(x_{0}\right)=C\left(x_{0}\right)$ for all $g \in \mathcal{A}$. As $f \in \mathcal{B}$, by (6) we obtain $f\left(x_{0}\right)=C\left(x_{0}\right)$ and this contradicts (7). Therefore

$$
\text { for all } y \geq x_{0} \text { with } f_{0}(y)>f_{0}\left(x_{0}\right) \text { we have } C(y)=C\left(x_{0}\right) \text {. }
$$

Choose $y \geq 0$ satisfying (8); as $n y \geq y \geq x_{0}$ and $f_{0}(n y)=n f_{0}(y) \geq f_{0}(y)>$ $f_{0}\left(x_{0}\right)$ for all natural $n \geq 1$, condition (8) gives $n C(y)=C\left(x_{0}\right)$ for all $n \geq 1$. We obtain $C(y)=(n+1) C(y)-n C(y)=C\left(x_{0}\right)-C\left(x_{0}\right)=0$, and therefore $C\left(x_{0}\right)=C(y)=0$. As $f \in \mathcal{B}$, from (6) we deduce that $f\left(x_{0}\right)=0$, contrary to $(7)$.

REMARK. The $f_{0}$-relevance was essential in proving formula (8). The finite-dimensional examples in [2] show that this condition cannot be removed.

Proposition 3. Let $C$ be an $f_{0}$-relevant (o)-continuous cost function and $E^{\times}$a Banach subspace of $E^{\prime}$. Then $\mathcal{B} \subseteq \mathcal{A}$ implies $N W_{C} \subseteq U A_{C}$. 
Proof. According to Lemma 1 and our hypothesis we have $\mathcal{A}=\mathcal{B}$. In addition,

$$
\begin{aligned}
C(x) & =\inf \{C(y) ; y \geq x \text { and } C(y)+C(-y)=0\} \\
& =\min \{C(y), y \geq x \text { and } C(y)+C(-y)=0\} .
\end{aligned}
$$

Indeed, as in Lemma 1, we obtain $C(y)=g(y)$ for all $g \in \mathcal{A}=\mathcal{B}$ provided $C(y)+C(-y)=0$ and, using that $E^{\times}$is Banach, by the Krein-Šmulian theorem (see [8]) and the fact that $C(\cdot)$ is (o)-continuous, we find that $\mathcal{A}$ is weak*-compact and the inf in the first line of formula (9) is attained so that we are allowed to write "min".

Now let $x_{0} \in N W_{C}$; by (5) we consider $y \geq x_{0}$ with $f_{0}(y)>f_{0}\left(x_{0}\right)$ and such that $C(y)>C\left(x_{0}\right)$. If $C\left(x_{0}\right)+C\left(-x_{0}\right) \neq 0$, by (9) we obtain

$$
C\left(x_{0}\right)=\min \left\{C(y) ; y>x_{0} \text { and } C(y)+C(-y)=0\right\},
$$

so there exists $z>x_{0}$ with $C(z)+C(-z)=0$ and $C\left(x_{0}\right)=C(z)$. Therefore we should have $f_{0}(z)=f_{0}\left(x_{0}\right)$, which contradicts the assumption at the begining of the paragraph, so $C\left(x_{0}\right)+C\left(-x_{0}\right)=0$, and by (4), $x_{0} \in U A_{C}$.

REMARK. Both the (o)-continuity of the cost function and the technical restriction " $E^{\times} \subset E^{\prime}$ is a Banach subspace" were essential in proving formula (9). Examples 3.7 and 3.10 in [4] show that neither condition can be removed.

Proof of the Theorem. Apply Lemma 1 and Propositions 2-3.

\section{References}

[1] C. D. Aliprantis and O. Burkinshaw, Locally Solid Riesz Spaces with Applications to Economics, Amer. Math. Soc., Providence, 2003.

[2] A. Araujo, A. Chateauneuf and J.-H. Faro, Revealing incomplete financial markets, IMPA preprint, Rio de Janeiro, 2008.

[3] P. Artzner, F. Delbaen, J.-M. Eber and D. Heath, Coherent measures of risk, Math. Finance 9 (1999), 203-228.

[4] F. Delbaen, Coherent risk measures on general probability spaces, in: Advances in Finance and Stochastics, K. Sandmann et al. (eds.), Springer, Berlin, 2002, 1-37.

[5] H. Föllmer and A. Schied, Convex measures of risk and trading constraints, Finance Stoch. 6 (2002), 429-447.

[6] - - - Robust preferences and convex measures of risk, in: Advances in Finance and Stochastics, K. Sandmann et al. (eds.), Springer, Berlin, 2002, 39-56.

[7] M. Frittelli and E. R. Gianin, Putting order in risk measures, J. Banking Finance 26 (2002), 1473-1486.

[8] A. Grothendieck, Topological Vector Spaces, Gordon and Breach, Philadelphia, 1992.

[9] S. Jaschke and U. Küchler, Coherent risk measures and good-deal bounds, Finance Stoch. 5 (2001), 181-200.

[10] G. Stoica, Relevant coherent measures of risk, J. Math. Econom. 42 (2006), 794-806. 
[11] A. C. Zaanen, Introduction to Operator Theory in Riesz Spaces, Springer, Berlin, 1997.

G. Stoica

Department of Mathematical Sciences

University of New Brunswick

Saint John NB, E2L 4L5 Canada

E-mail: stoica@unb.ca

Received on 18.3.2010;

revised version on 8.6.2010

(2038) 
